\title{
Abstract \\ Responsive feeding practices among caregivers in rural Anuradhapura; a missing component in infant feeding! \\ Senevirathne $\mathrm{SS}^{1 *}$, Karunamuni ${ }^{1}$, Pallewaththa PWPWK ${ }^{1}$, Rathnayake $\mathrm{RMM}^{1}$, Wickramage $\mathrm{K}^{2}$, Agampodi $\mathrm{SB}^{1}$, Agampodi TC ${ }^{1}$ \\ ${ }^{1}$ Maternal and Child Health Research Unit, Department of Community Medicine, Faculty of Medicine and Allied Sciences, Rajarata University of Sri Lanka \\ ${ }^{2}$ International Organization for Migration, Colombo, Sri Lanka
}

\begin{abstract}
Background

Responsive feeding (RF) is a strategy that has a positive impact on growth, caregiver behaviours and dietary intake of children. But concern given to RF in national nutrition programmes is inadequate. Our aim is to explore the knowledge and practices on RF among mothers having infants of 9 months in Nuwaragam Palatha Central (NPC) $\mathrm{MOH}$ area, Anuradhapura.

\section{Methods}

We conducted a qualitative study using in-depth interviews with mothers who were purposefully selected to represent different socio-demographic backgrounds. In-depth interviews were conducted according to Family Health International guidelines. Data analysis was done using framework approach. Ethical clearance was obtained for the study.

Results

Though mothers practice some components of the four step approach of RF, none of them seem to have effectively practiced the full concept in feeding their child. Only few mothers have prepared a separate feeding place. But, even those mothers were unable to stick to that place. And the preparation of the place was not satisfactory to meet the child's desires. Mothers were good at identifying hunger and satiety signs. However, majority of mothers were not feeding their children in response to hunger signs but according to a time table made on their own. Letting the child watch television and taking the child around the garden were major factors of distraction while feeding. Mothers stated the difficulty in introducing new food to their children indicating the lack of reciprocity between the child and the caregiver.

\section{Conclusions}

The concept of RF is not effectively practiced in the community of NPC, MOH area.
\end{abstract}

Key words: Responsive Feeding practices; Care givers; Rural Anuradhapura

Copyright: (C) 2015 Senevirathne SS et al. This is an open access article distributed under the Creative Commons Attribution License, which permits unrestricted use, distribution, and reproduction in any medium, provided the original work is properly cited.

* Correspondence : seegirisri@gmail.com

Cite this aabstract as: Senevirathne SS, Karunamuni N, Pallewaththa PWPWK, Rathnayake RMM, Wickramage K, Agampodi SB, Agampodi TC.Responsive feeding practices among caregivers in rural Anuradhapura; a missing component in infant feeding!.Anuradhapura Medical Journal 2015;9 (2Supp):S04.

DOI: http://dx.doi.org/10.4038/amj.v9i2Supp.7553 
